\title{
Dopamine transporter occupancy by methylphenidate and impulsivity in adult ADHD
}

\author{
Cleo L. Crunelle, Wim van den Brink, Geert Dom and Jan Booij
}

\begin{abstract}
Summary
Impulsivity is a multidimensional construct, including impulsive decision-making and impulsive action, representing relatively independent neurocircuitries. ADHD is treated with methylphenidate, a drug that binds to dopamine transporters. This study in 24 adult male patients with ADHD shows that dopamine transporter occupancy by methylphenidate in the putamen correlates with improvements in cognitive but not in motor impulsivity.
\end{abstract}

\section{Declaration of interest}

W.v.d.B. received fees from Eli Lilly unrelated to the present work. G.D. received fees from Lundbeck, Janssen Pharmaceuticals and Eli Lilly unrelated to the present work. J.B. has received consultancy payments from GE Healthcare.
Impulsivity, a key symptom and characteristic of attention-deficit hyperactivity disorder (ADHD), is a multifactorial construct including motor impulsivity and cognitive impulsivity. ${ }^{1}$ Although motor and hyperactivity symptoms seem to decline with progression into adulthood, ${ }^{2}$ cognitive impulsivity (i.e. impulsive decisionmaking) remains prominent in adult ADHD and is associated with a range of clinical consequences (e.g. poor relational or professional stability). A recent study reported that impulsivity was positively associated with the availability of striatal dopamine transporters, ${ }^{3}$ although this was not replicated in a larger study. ${ }^{4}$ In adults with ADHD, increased dopamine transporter availability was observed, but a recent meta-analysis suggested that this increase was driven by patients that were previously medicated. ${ }^{5}$ Methylphenidate widely prescribed for the treatment of childhood ADHD but more debated as to its use in adult populations - binds to dopamine transporters and reduces symptoms of ADHD. However, there is no direct evidence that dopamine transporter occupancy by methylphenidate is associated with a reduction of impulsivity symptoms in the cognitive and/or motor domains of impulsivity. In this study, we investigated whether striatal dopamine transporter occupancy by methylphenidate is related to improvements in the distinct domains of cognitive and motor impulsivity.

\section{Method}

A total of 24 male medication-naive adult patients with ADHD were included in the study. Recruitment, diagnosis and inclusion/ exclusion criteria are described elsewhere (trial registered with Nederlands Trial Register, NTR3127). ${ }^{6}$ Eight patients had a comorbid diagnosis of cocaine dependence and were abstinent for $>2$ weeks prior to inclusion and no other psychiatric comorbidities were present. Striatal dopamine transporter availability and impulsivity levels were assessed at baseline (before medication) and after a 2-week treatment with extended-release methylphenidate (MPH-OROS $54 \mathrm{mg}$ ). Dopamine transporter availability $\left(\mathrm{BP}_{\mathrm{ND}}\right)$ was assessed on a brain-dedicated single-photon emission computed tomography system using the caudate and putamen as regions of interest. ${ }^{7}$ A structural magnetic resonance imaging scan was made of each patient for co-registration. On the last treatment day, $\mathrm{BP}_{\mathrm{ND}}$ was assessed $5 \mathrm{~h}$ after supervised intake of the last methylphenidate tablet and dopamine transporter occupancy by methylphenidate was calculated as previously described. ${ }^{6}$ Cognitive and motor impulsivity were assessed using a delayed discounting task and a stop-signal task respectively. The outcome measures were the discounting rate $k$ as a measure of cognitive impulsivity (where higher $k$ values represent higher cognitive impulsivity ${ }^{8}$ ) and the Stop Signal Reaction Time $(\text { SSRT })^{9}$ as a measure of speed of inhibition to assess motor impulsivity. Impulsivity was measured at baseline and reassessed on the last day in the hour prior to dopamine transporter imaging. Improvement in impulsivity measures were calculated relative to baseline. The study was approved by the ethical committee of the Academic Medical Centre of the University of Amsterdam.

Statistics were performed using SPSS version 20 for Windows. Changes in impulsivity following methylphenidate were analysed using paired sample $t$-tests. Linear regression analyses were performed to assess the relationship between dopamine transporter occupancy by methylphenidate in striatal regions (dependent variable) and improvements in primary outcome measures on impulsivity tasks following methylphenidate (independent variables). Analyses were carried out separately for caudate and putamen (mean left and right sides). Separate analyses were performed for patients with ADHD with and without a comorbid diagnosis of cocaine dependence. An alpha of 0.05 was set as statistically significant.

\section{Results}

Our entire sample was male, 34 (s.d. =6) years old, had a mean of 10 (s.d. =4) years of education and an IQ of 105 (s.d.=5). Body mass index was $26 \mathrm{~kg} / \mathrm{m}^{2}$ (s.d.=4). One patient was diagnosed with the hyperactive ADHD subtype, 10 with the inattentive subtype and 13 with combined ADHD subtype. Patients had a mean ADHD Symptom Rating Scale score of 12 (s.d.=4), indicating substantial severity of ADHD symptoms. ${ }^{10}$ Eleven patients were smokers and had a nicotine dependency score of $5($ s.d. $=2)$ on the Fagerström Test for Nicotine Dependence. ${ }^{11}$ Patients scored 82 (s.d.=13) on the Barratt Impulsiveness Scale, indicating substantial impulsivity. ${ }^{12}$

Following methylphenidate, $\mathrm{BP}_{\mathrm{ND}}$ decreased with $33 \%$ $($ s.d. $=16)$ and $41 \%($ s.d. $=12)$ in the putamen and caudate nucleus respectively, indicating substantial dopamine transporter occupancy by methylphenidate. Following methylphenidate treatment, the discounting rate $k$ decreased significantly $(t=-2.31 ;$ d.f. $=23 ; P=0.030)$ and this decrease correlated significantly with dopamine transporter occupancy by methylphenidate in the putamen $(B=618.71 ; t=2.98 ; P=0.010)$, but not in the caudate $(P=0.460$; not significant). The SSRT also decreased significantly following methylphenidate treatment $(t=-2.46$; d.f. $=23 ; P=0.023)$, but changes were not correlated with dopamine transporter occupancy by methylphenidate in putamen or caudate (both $P>0.389$; not significant). In an 
exploratory analysis, we found that the correlation of dopamine transporter occupancy by methylphenidate with the discounting rate $k$ (in the putamen) was stronger in patients without cocaine dependence $(B=4435.27 ; t=4.38 ; P=0.001)$ than in those with cocaine dependence $(B=495.23 ; t=2.53 ; P=0.045$; online Fig. DS1). No other correlations were observed between dopamine transporter occupancy by methylphenidate in putamen/caudate nucleus with cognitive or motor impulsivity measures for subgroups of patients with or without cocaine dependence (all $P>0.267$; not significant).

\section{Discussion}

This report provides preliminary evidence that dopamine transporter occupancy by methylphenidate in the putamen drives improvements in cognitive impulsivity in male adult patients with $\mathrm{ADHD}$, i.e. that a higher occupancy of dopamine transporters by methylphenidate leads to better cognitive impulsivity. In contrast, motor impulsivity was not correlated with dopamine transporter occupancy by methylphenidate in putamen or caudate. Although the putamen is traditionally associated with the 'motor loop' of the corticobasal gangliathalamic circuit, more recent studies also suggest a role of the putamen in salience pathways (i.e. orbitofrontal cortex, insula, anterior cingulate gyrus), especially in patients with ADHD. ${ }^{13,14}$ Yu suggested an additional pathway within patients with ADHD connecting left insula and left anterior cingulate gyrus via frontal gyrus and putamen. ${ }^{14}$ Through this pathway, changes in putamen function may modulate salience behaviour, including delay discounting. Our findings are in line with this hypothesis, but clearly need replication.

In previous studies in adult $\mathrm{ADHD}$, dopamine transporter occupancy by methylphenidate $(3 \times 5 \mathrm{mg})$ ranges between 29 and $51 \%,{ }^{15}$ with wide variability in dopamine transporter occupancy from one dose. One explanation for this variability may be differences in body weight between participants, while the methylphenidate dose was not weight-corrected. However, post hoc, we did not find a statistically significant association between body weight and dopamine transporter occupancy $(P>0.115)$. It is also likely that there will be differences between patients in the rate they can metabolise methylphenidate (fast $v$. slow). Also, previous ADHD treatments as well as the severity of ADHD symptoms may lead to conformational changes in the dopamine transporter, and consequently to changes in dopamine transporter occupancy by methylphenidate. Further studies may test these postulates.

Clinically, deficits in cognitive impulsivity have devastating effects on a patient's life choices (i.e. risk-taking) and life quality. Our data suggest a benefit of methylphenidate specifically on these impulsive choice mechanisms in adult patients with ADHD. These findings, although in need of replication, provide a new element in the ongoing debate on stimulant prescription in the treatment of adult ADHD. ${ }^{16}$ Our findings suggest that methylphenidate specifically improves one domain of impulsivity in ADHD (the cognitive domain), and that (a) perhaps other pharmacotherapy might be more effective in tempering the motor impulsivity symptoms in ADHD or that (b) methylphenidate improves the motor impulsivity domain through a mechanism other than occupancy of striatal dopamine transporters.

Strengths of this study include the assessment of dopamine transporter and separate domains of impulsivity in a single patient population and the assessments at separate time points before and after methylphenidate treatment. The impulsivity tasks are well validated and widely used in clinical and preclinical settings, where they have shown to represent important, but largely unrelated, aspects of impulsivity. ${ }^{1}$ The main limitation of this study is the small sample size. Future studies should investigate whether methylphenidate treatment correlates with improved functional connectivity linking striatal and frontal brain regions (e.g. using functional magnetic resonance imaging) and whether these findings can be validated in larger (ADHD clinical and non-clinical) populations.

\begin{abstract}
Cleo L. Crunelle, PhD, Collaborative Antwerp Psychiatric Research Institute (CAPRI) University of Antwerp, Belgium; Amsterdam Institute for Addiction Research and Departments of Psychiatry and Nuclear Medicine, Academic Medical Center, University of Amsterdam, The Netherlands; Wim van den Brink, MD, PhD, Amsterdam Institute for Addiction Research and Department of Psychiatry, Academic Medical Center, University of Amsterdam, The Netherlands; Geert Dom, MD, PhD, Collaborative Antwerp Psychiatric Research Institute (CAPRI), University of Antwerp, and Psychiatric Centre Alexian Brothers, Boechout, Belgium; Jan Booij, MD, PhD, and Psychiatric Centre Alexian Brothers, Boechout, Belgium; Jan Booij, MD,
Department of Nuclear Medicine, Academic Medical Center, University of Department of Nuclear Medicine,
Amsterdam, The Netherlands
\end{abstract}

Correspondence: C. L. Crunelle, Collaborative Antwerp Psychiatric Research Institute (CAPRI) - University of Antwerp, Campus Drie Eiken, Universiteitsplein 1-R.3.27, 2610 Antwerp (Wilrijk), Belgium. Email: cleo.crunelle@gmail.com

First received 29 May 2013, final revision 29 Nov 2013, accepted 10 Feb 2014

\section{Funding}

Grant to W.v.d.B. from ZonMW.

\section{References}

1 Broos N, Schmaal L, Wiskerke J, Kostelijk L, Lam T, Stoop N, et al. The relationship between impulsive choice and impulsive action: a cross-species translational study. PLOS One 2012; 7: e36781.

2 Biederman J, Petty CR, Evans M, Small J, Faraone SV. How persistent is ADHD? A controlled 10-year follow-up study of boys with ADHD. Psychiatry Res 2010; 177: 299-304.

3 Costa A, la Fougère C, Pogarell O, Möller HJ, Riedel M, Ettinger U. Impulsivity is related to striatal dopamine transporter availability in healthy males. Psychiatry Res 2013; 211: 251-6.

4 Burke SM, van de Giessen E, de Win M, Schilt T, van Herk M, van den Brink W, et al. Serotonin and dopamine transporters in relation to neuropsychological functioning, personality traits and mood in young adult healthy subjects. Psychol Med 2012; 41: 419-29.

5 Fusar-Poli P, Rubia K, Rossi G, Sartori G, Balottin U. Striatal dopamine transporter alterations in ADHD: pathophysiology or adaptation to psychostimulants? A meta-analysis. Am J Psychiatry 2013; 169: 264-72.

6 Crunelle CL, van den Brink W, veltman DJ, van Emmerik-van Oortmerssen K, Dom G, Schoevers RA, et al. Low dopamine transporter occupancy by methylphenidate as a possible reason for reduced treatment effectiveness in ADHD patients with cocaine dependence. Eur Neuropsychopharmacol 2013; 23: $1714-23$.

7 Crunelle CL, Veltman DJ, van Emmerik-van Oortmerssen K, Booij J, van den Brink W. Impulsivity in adult ADHD patients with and without cocaine dependence. Drug Alcohol Depend 2013; 129: 18-24.

8 Bickel WK, Marsch LA. Toward a behavioral economic understanding of drug dependence: delay discounting processes. Addiction 2001; 96: 73-86.

9 Logan GD, Cowan WB, Davis KA. On the ability to inhibit simple and choice reaction time responses: a model and a method. J Exp Psychol Hum Percept Perform 1984; 10: 276-91.

10 Kooij JJ, Buitelaar JK, van den Oord EJ, Furer JW, Rijnders CA, Hodiamont PP. Internal and external validity of attention-deficit hyperactivity disorder in a population-based sample of adults. Psychol Med 2005; 35: 817-27.

11 Heatherton TF, Kozlowski LT, Frecker RC, Fagerström KO. The Fagerström Test for Nicotine Dependence: a revision of the Fagerström Tolerance Questionnaire. Br J Addict 1991; 86: 1119-27.

12 Patton JH, Stanford MS, Barratt ES. Factor structure of the Barratt impulsiveness scale. J Clin Psychol 1995; 51: 768-74.

13 Wilbertz G, van Elst LT, Delgado MR, Maier S, Feige B, Philipsen A, et al. Orbitofrontal reward sensitivity and impulsivity in adult attention deficit hyperactivity disorder. Neurolmage 2012; 60: 353-61.

14 Yu D. Additional brain functional network in adults with attention-deficit/ hyperactivity disorder: a phase synchrony analysis. PLOS One 2013; 8: e54516.

15 Krause J. SPECT and PET of the dopamine transporter in attention-deficit/ hyperactivity disorder. Expert Rev Neurother 2008; 8: 611-25.

16 Ramos-Quiroga JA, Montoya A, Kutzelnigg A, Deberdt W, Sobanski E. Attention deficit hyperactivity disorder in the European adult population: prevalence, disease awareness, and treatment guidelines. Curr Med Res Opin 2013; 29: 1093-104. 